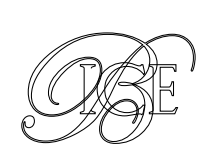

José Pedro Sebastián de Erice*

Pablo V. Rovetta Dubinsky**

\title{
LA RUTA DE LA SEDA Y LA COOPERACIÓN EN TERCEROS MERCADOS DESDE LA PERSPECTIVA EMPRESARIAL. EL CASO DE TÉCNICAS REUNIDAS
}

El presente artículo hace una breve presentación de Técnicas Reunidas, SA, su experiencia en China y con empresas chinas en terceros países. El trabajo hace una mención especial a la iniciativa de «la Franja y la Ruta» planteada por el presidente Xi Jinping, más conocida por sus siglas en inglés, OBOR. Según los autores, España y su Administración no solo están en el OBOR — cosa que en algunos sectores se duda-, sino que llevan realizando importantes esfuerzos con el fin de que las empresas españolas puedan beneficiarse de esta iniciativa.

Palabras clave: OBOR, Banco Asiático de Inversiones en Infraestructuras (AIIB), rutas de la seda, «la Franja y la Ruta».

Clasificación JEL: F23, G38.

\section{Técnicas Reunidas, SA}

Técnicas Reunidas, SA es una empresa española dedicada al diseño y construcción de plantas industriales relacionadas con el petróleo, el gas, la petroquímica y la generación de electricidad, en concreto lo que se conoce como plantas llave en mano.

En 1968 comenzamos nuestra andadura internacional y ya en 1981 más de la mitad de nuestros ingresos provenían de fuera de España. Actualmente el 100 por 100 de la facturación de Técnicas Reunidas se produce en el exterior y una parte muy importante de ella, de

\footnotetext{
*Secretario General de Técnicas Reunidas, SA.

**Director para China en Técnicas Reunidas, SA.

Versión de diciembre de 2017.
}

los países que forman la llamada «una Franja, una Ruta», de la cual hablaremos más adelante.

En concreto, desde el año 2003 es en la zona de Arabia Saudí y los países de la zona del Golfo donde estamos realizando una parte muy importante de nuestros proyectos, aunque trabajamos en los cinco continentes.

\section{Técnicas Reunidas, SA y China}

Técnicas Reunidas comenzó sus actividades de promoción comercial en China a principios de los años ochenta del siglo pasado.

Con una importante ayuda política y financiera de la Administración española, al igual que la que esta brindaba a las empresas que $D$ 
José Pedro Sebastián de Erice y Pablo V. Rovetta Dubinsky

empezaban a entrar en ese mercado, en 1988, o sea, treinta años atrás, firmamos nuestro primer contrato en China. En 1991 establecimos una oficina permanente en Pekín. Hemos firmado un total de veintiséis contratos y trabajado para las tres principales empresas de petróleo del país (CNPC-Petrochina, SINOPEC y CNOOC), así como para varias multinacionales del sector en proyectos de empresas mixtas con socios chinos o también para empresas extranjeras con capital propio. Entre los clientes extranjeros para los cuales trabajamos se encuentran empresas de la importancia de BASF, Shell, ExxonMobil, Saudi Aramco o CEPSA.

\section{La Franja y la Ruta}

En el año 2013, el presidente chino Xi Jinping lanzó la iniciativa de «la Franja y la Ruta», conocida también por sus nombres en inglés como Belt and Road o One Belt One Road -OBOR - con el objetivo de restablecer dos rutas comerciales de China con el exterior de mucha importancia en el pasado: la llamada «Ruta de la Seda» y la «Ruta de la Seda por Mar».

La antigua Ruta de la Seda fue un movimiento comercial que tuvo lugar hace más de dos mil años, que unía la ciudad de Xi'an (literalmente Paz del Oeste), conocida entonces como Chang'an (Paz Eterna), con los países de Asia Central, Oriente Medio y África, llegando hasta las puertas de lo que hoy es Europa Occidental.

Xi'an fue la capital cuando China se unificó por primera vez durante la dinastía Qin (221206 a.C.) y posteriormente durante la dinastía Han (206 a.C. - 220 de nuestra era), que fue cuando comenzaron los contactos comerciales con el resto del mundo, en lo que se conoció como la Ruta de la Seda. Aunque el comercio abarcaba todo tipo de productos, la seda china fue uno de los más apreciados, y de ahí el nombre de esa ruta.

Por otro lado, alrededor de quinientos años antes de que Colón descubriera América, un almirante chino llamado Zheng He realizó siete expediciones que pasaban por el Pacífico, el Índico y llegaban hasta la costa del este de África. Esas expediciones, formadas por cientos de barcos, se dedicaban al comercio, intercambiando productos chinos por los de los territorios a los cuales llegaban. Un hecho a destacar es que esas expediciones al Pacífico eran puramente comerciales, sin enfrentamientos militares, y sin que China ocupara ninguna parte de los territorios a los cuales Zheng $\mathrm{He}$ iba llegando.

Aunque por parte china no se menciona esto directamente cuando se habla de la Franja y la Ruta, hubo otro movimiento comercial global que unió a Filipinas (a donde llegaban productos desde China y otros países asiáticos) con lo que hoy es México, pasando por España, a través de un barco conocido como El Galeón de Manila o La Nao de China. De ahí viene precisamente el famoso «mantón de Manila», que en realidad era chino, y de seda.

En todo caso, las iniciativas planteadas por el presidente Xi Jinping y su Gobierno a partir del 2013 hablan de lo que podíamos llamar «dos rutas de la seda», y por eso la parte china la llama una Franja, una Ruta. En este sentido, consideramos que no es tan conveniente hablar de «la nueva Ruta de la Seda» como referirse a «las nuevas rutas de la seda», al nombre español que usa el Gobierno chino «la Franja y la Ruta» o a las más conocidas siglas ya citadas de OBOR.

Desde el punto de vista geográfico, hay decenas de mapas que indicarían los territorios por donde pasarían esas dos rutas de la $D$ 


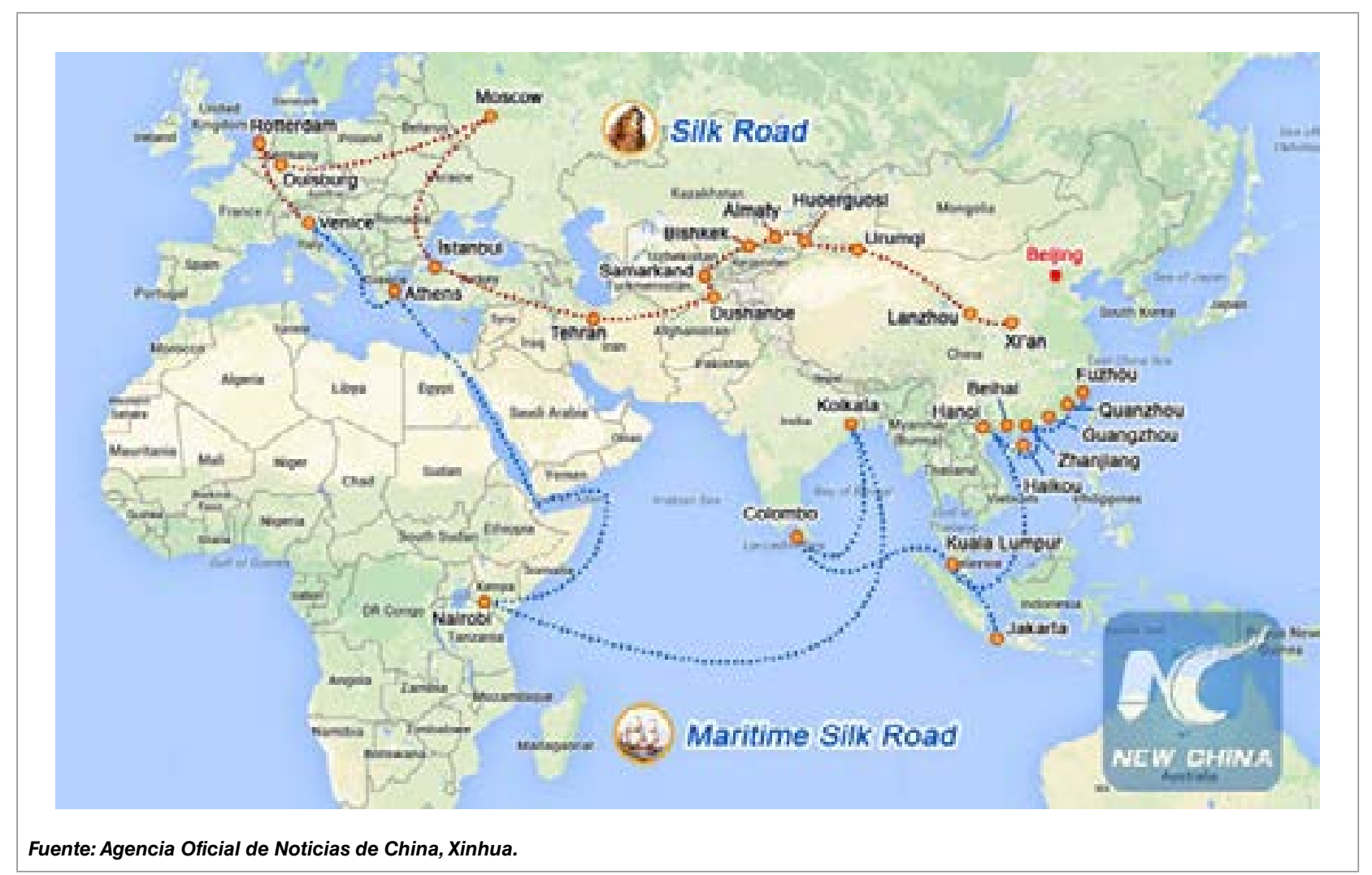

seda. Muchos de esos mapas son contradictorios, en especial en lo que respecta a los países que abarcarían estas dos rutas, y, por eso, hemos preferido usar uno de la Agencia Oficial de Noticias de China, Xinhua, como el que se muestra en esta página.

Si tomáramos como referencia este mapa oficial, España no estaría en las rutas de la seda. Sin embargo, el concepto de «una Franja, una Ruta» se ha ido ampliando con el paso del tiempo. Su principal objetivo original era establecer importantes obras de infraestructuras para facilitar el comercio entre China, los países de Asia Central, el este de África, Oriente Medio, el sur del Mediterráneo (a través de Grecia) y parte del continente europeo, en particular Europa Central.

Sin embargo, paulatinamente, el ámbito geográfico se fue ampliando, China ha ido invitando a un número cada vez mayor de países a participar en ese proyecto que, sin exagerar, prácticamente pretende abarcar gran parte del mundo.

Un ejemplo de la ampliación geopolítica de esta iniciativa es la presencia de la presidenta de Chile, Michelle Bachelet, en el Foro de la Franja y la Ruta que tuvo lugar en Pekín en mayo de 2017. Otro ejemplo más se dio en el mes de noviembre de 2017, cuando el presidente de Panamá, Juan Carlos Varela, visitó Pekín y trató con el presidente Xi Jinping la participación del país centroamericano en las rutas de la seda.

Por lo tanto, y a pesar de su nombre, la iniciativa del presidente Xi Jinping y de su Gobierno intenta abarcar el máximo de países y crear las condiciones para incrementar al máximo posible el comercio de China con el resto del mundo, así como la presencia de empresas chinas a nivel global. 
José Pedro Sebastián de Erice y Pablo V. Rovetta Dubinsky

Para lo relacionado con los antecedentes y los objetivos de las rutas de la seda nos permitimos recomendar el excelente artículo escrito por Javier Serra Guevara en el Boletín Económico de ICE, n. 3072 , correspondiente al mes de enero de 2016, del cual figuran en este artículo algunos datos o conceptos.

\section{España en las rutas de la seda}

Desde un comienzo, la Administración española mostró su interés por este proyecto lanzado por Xi Jinping y ha realizado innumerables esfuerzos para que las empresas españolas puedan participar en el mismo.

La mejor prueba de ello es la presencia del presidente del Gobierno español, Mariano Rajoy, el 20 de mayo del 2017 en el Foro de la Franja y la Ruta celebrado en Pekín. Posteriormente, el rey de España tuvo oportunidad en Astana de tratar este tema con el presidente chino Xi Jinping, reiterando el compromiso de España ante esta iniciativa.

Aparte de ello, desde la Secretaría de Estado de Comercio, el ICEX y la Oficina Comercial de España en Pekín, se han tomado diversas iniciativas tanto en España como en China. La última de ellas ha sido el Foro España-China en terceros mercados. Oportunidades de cooperación en equipamiento, ingeniería e infraestructuras, que tuvo lugar en Pekín del 18 al 20 de diciembre. El foro fue organizado conjuntamente con la National Development and Reform Comission (NDRC) y contó con una amplia representación de empresas chinas, así como con un selecto grupo de empresas españolas. Durante el foro, aparte de las reuniones bilaterales entre empresas, tuvieron lugar presentaciones de empresas españolas y chinas sobre sus experiencias en terceros mercados.
También formó parte muy importante de este foro las visitas organizadas al Banco Asiático de Inversiones en Infraestructuras (conocido por sus siglas en inglés como AllB) y a la empresa Sinosure (el equivalente al CESCE español), así como la participación de instituciones como el China Development Bank o el China Exim Bank. Este es un elemento fundamental ya que la financiación es uno de los aspectos más importantes de «la Franja y la Ruta».

A estos efectos, y bajo la iniciativa del Gobierno chino, se estableció en Pekín el AllB. En dicho banco se dejó un porcentaje del 25 por 100 para países no asiáticos y España tuvo el acierto de participar desde un principio, con un fondo de 350 millones de dólares, convirtiéndose en miembro fundador del banco, que comenzó oficialmente a funcionar en enero de 2016. En un principio, el AllB contaba con 57 miembros, entre ellos España, como hemos indicado, y ahora ya ha llegado a la cifra de 84.

El actual ministro de Economía, Industria y Competitividad del Gobierno de España, Luis de Guindos, es, por parte española, gobernador del AllB.

Por lo tanto, y ante las dudas que algunas veces se plantean de forma pública y privada, es necesario recordar y confirmar que España está, desde el principio, en el proyecto de «una Franja, una Ruta».

\section{La experiencia de Técnicas Reunidas en la Franja y la Ruta}

Tras décadas de experiencia trabajando en China, conociendo sus ingenierías, sus empresas de construcción y sus métodos de trabajo, Técnicas Reunidas tomó la decisión, desde comienzos del presente siglo, de empezar a $\triangleright$ 
trabajar con empresas chinas en terceros mercados. Pasamos entonces a una nueva etapa estratégica en nuestra relación con China. En concreto, de «Técnicas Reunidas en China» comenzamos a emplear el enfoque de «écnicas Reunidas y China» o «Técnicas Reunidas con China».

El primer proyecto en el cual trabajamos juntos lo firmamos en el año 2005, y fue para la construcción de una refinería en Arabia Saudí. Por lo tanto, llevamos más de doce años trabajando con empresas chinas en terceros países.

Las constructoras chinas actuaron en ese megaproyecto como subcontratistas de Técnicas Reunidas en construcción y montaje. La experiencia positiva de este primer proyecto nos llevó a continuar trabajando con empresas de construcción chinas principalmente en Arabia Saudí, pero también en otros mercados como Malasia o Kuwait.

A mediados de 2015, sin embargo, se produjo un salto cualitativo en nuestras relaciones con empresas chinas en terceros países. En concreto, a mediados de ese año, Técnicas Reunidas formó un consorcio con la empresa Sinopec Engineering Group (SEG) y la coreana Hanwha Engineering \& Construction (esta última con una participación minoritaria), presentó una oferta y se adjudicó el diseño y la construcción en base «llave en mano» de las unidades de proceso -las más importantesde la nueva refinería Al-Zour en Kuwait, para la Kuwait National Petroleum Company (KNPC), que será una de las más grandes de Oriente Medio. El contrato tiene un valor aproximado de 4.100 millones de dólares, y Técnicas Reunidas es el líder del mismo, en el cual tiene una participación del 51 por 100 .

Como resumen, entonces, Técnicas Reunidas ha estado y está trabajando en un total de doce proyectos con empresas chinas en terceros países, incluyendo Arabia Saudí, Bolivia, Kuwait y Malasia.

Como resultado de todos estos contratos, el monto total de los trabajos subcontratados a empresas chinas en terceros países, más la participación china en el proyecto de Kuwait, supera la cifra de 5.000 millones de dólares.

Por lo tanto, siendo una empresa netamente internacional, donde el cien por cien de nuestros ingresos provienen del mercado exterior, habiendo tenido una experiencia de más de doce años trabajando con empresas chinas en terceros países, y cerca de treinta en China, no podemos dejar de prestar atención a la iniciativa de la Franja y la Ruta, y hacer los máximos esfuerzos para intentar obtener oportunidades de negocio en la misma, junto con empresas chinas.

Si bien una gran parte de las inversiones en la Franja y la Ruta serán en el sector de las infraestructuras (carreteras, puentes, túneles, puertos, etcétera), el cual está fuera de nuestras áreas de actividad, consideraremos e intentaremos crear oportunidades en sectores de nuestro interés como la energía -muchos de los países de Asia Central, por ejemplo, son muy ricos en recursos energéticos-.

En la reunión con el AllB que acertadamente se organizó en diciembre sobre «EspañaChina en terceros países» se nos indicó que uno de los objetivos del banco es y será la inversión en infraestructuras y «otros sectores productivos", y es ahí donde podríamos tener oportunidades de negocio.

Al mismo tiempo, somos conscientes de que la influencia política de China con la mayor parte de los países de Asia Central es muy estrecha y sus relaciones económicas son muy dilatadas y fuertes. A la vez, la presencia empresarial de China en Asia Central, el Sudeste Asiático o África es $\triangleright$ 
también muy amplia. Por último, las enormes posibilidades de financiación que tiene China -que aparte de ser el accionista mayoritario del AIIB también ha creado un Fondo de la Ruta de la Seda, dotado de 40.000 millones de dólares - de alguna manera nos llevará a tener que unirnos con empresas chinas para realizar proyectos que se incluyan en esta iniciativa, ya que las posibilidades de competir solos con empresas chinas, en principio, no parecen muy realistas.

Teniendo en cuenta nuestra experiencia, que no necesariamente es extrapolable a otras empresas, consideramos que es importante, en nuestra opinión, y se estará en una mejor posición si se pueden cumplir por los menos con las siguientes tres condiciones:

1. Las empresas españolas que llevan años instaladas en China y que tienen experiencia de trabajo con compañías chinas, como es el caso de Técnicas Reunidas entre otras, estarán mejor posicionadas para trabajar juntas en terceros países. El conocimiento de las empresas chinas, la experiencia alcanzada con ellas, la comprensión de su forma de pensar y de actuar son condiciones que pueden ayudar a trabajar juntos.

2. La complementariedad entre las partes española y china o la envergadura de los proyectos - que si son muy grandes requieren uniones para disminuir riesgos y garantizar a los clientes grupos fuertes y con capacidad para encarar los proyectos- son también factores muy importantes a la hora de realizar obras en conjunto en terceros países.

3. La posibilidad de que las empresas españolas puedan participar en proyectos financiados por el AllB, el Fondo de la
Ruta de Seda u otras fuentes de financiación china y de que exista un marco transparente y claro sobre el funcionamiento del AllB también es un factor importante para que podamos participar juntos en proyectos del OBOR. Hay que tener en cuenta que nuestra competencia europea, o si queremos definirlo mejor, occidental, es y será muy fuerte, y en este sentido, consideramos que es muy importante el papel que pueda jugar la Administración española en el AllB.

El reto al cual nos enfrentamos no es fácil. Podríamos decir incluso que es mucho más difícil que el reto al que tuvimos que enfrentarnos en el mercado chino. Si en este teníamos $y$ tenemos diferencias culturales «bilaterales», y tuvimos que hacer un gran esfuerzo para conocer cómo funcionaba el país, sus normas, su Administración y su forma de trabajar; en el caso de un trabajo conjunto con empresas chinas en terceros países tenemos que combinar no dos, sino por lo menos tres elementos. A las diferencias culturales entre España y China hay que agregar las del país en el cual se realizará el proyecto, y esto mismo se aplica a sus costumbres, su Administración, sus normas, su forma de trabajar y un largo etcétera.

La experiencia de Técnicas Reunidas demuestra, en nuestra opinión, que es viable y puede ser beneficioso para ambas partes trabajar juntos en terceros países, pero también es probable que las dificultades se incrementen. Por ejemplo, un tema nuevo para nosotros fue, en temas de construcción, que tuvimos que enfrentarnos al problema de los visados para miles de trabajadores chinos, tema que se solucionó gracias a las buenas relaciones de China con los países en los $\triangleright$ 
cuales hemos trabajado. Esa posibilidad de llevar a miles de obreros chinos a trabajar en el exterior no es un caso que se pueda aplicar a todos los países, por lo que habrá que agregar un elemento más, y es el de trabajar con empresas de construcción locales, lo que incrementa las dificultades y los retos de comunicación y coordinación.

También hay que acostumbrarse a trabajar en, como mínimo, tres centros de operaciones: España, China y el país donde realizamos el proyecto (mientras que en China trabajábamos solo en dos); lo cual requiere más viajes, más y mejor coordinación, más y mejores comunicaciones; tres, y no dos, diferencias horarias; un dominio perfecto del inglés, que es la lengua común de trabajo, pero que para las tres partes (la unión española-china y el cliente) no deja de ser una lengua extranjera.

Es fundamental tener previstos los problemas de todo tipo que puedan surgir, tratar de resolverlos a medida que vayan surgiendo y no dejar pasar el tiempo y evitar que estos se conviertan en problemas más graves. También hay que tener en cuenta que China está cambiando de forma muy rápida, y que una experiencia o modo de trabajo de hace unos años atrás, o en un mercado determinado, ya no necesariamente pueden ser válidos en el presente o en otro mercado.

Un hecho que puede ser anecdótico, pero que es importante en la realización de estos proyectos que pueden llevar años de ejecución, es el Año Nuevo Chino, durante el cual se puede dar el caso de que sus trabajadores vuelvan a su país por un periodo mínimo de dos o tres semanas.

También un hecho importante a tener en cuenta es que con el desarrollo de la industria china y el incremento del nivel de vida de su población, y el de sus trabajadores en particular, los costes no necesariamente serán tan competitivos como antes.

Hemos expuesto las dificultades que pueden existir trabajando con empresas chinas en terceros países, pero, como ya hemos indicado, para nosotros no existen ni mercados ni proyectos «fáciles», y la iniciativa de trabajar junto con empresas chinas en terceros mercados es un hecho positivo que puede brindarnos nuevas oportunidades de negocio, de abrir nuevos mercados y/o consolidar nuestra posición en donde ya estamos presentes, siempre y cuando sea beneficioso para ambas partes y pueda haber una complementariedad mutua.

En definitiva, nuestra experiencia de trabajo con empresas chinas en terceros países ha sido en general positiva. También ha sido positiva para Técnicas Reunidas en mercados del OBOR, donde hemos trabajado y estamos trabajando solos o en unión con otras empresas no chinas, como por ejemplo en Rusia, Bangladés, Vietnam, Indonesia, Malasia y otros países de Oriente Medio como los Emiratos Árabes Unidos, Omán, Bahréin o Kuwait, entre otros.

Aparte de trabajar con empresas chinas, consideramos importante la posibilidad de trabajar para empresas chinas, en este caso de nuestro sector, que están ya y seguirán realizando inversiones en el exterior. Evidentemente, esas inversiones de empresas chinas en el exterior contarán con la participación de sus ingenierías, o empresas de construcción - cuando ello sea posible por las leyes del país donde se trabaja-, pero también empresas como Técnicas Reunidas —en unión con empresas chinas- pueden aportar su experiencia y know-how internacional, lo cual puede ser beneficioso para los inversores chinos en algunos países determinados.

En todo caso, consideramos que no podemos «perder el tren», que la presencia de $D$ 
José Pedro Sebastián de Erice y Pablo V. Rovetta Dubinsky

empresas chinas en terceros países es cada vez mayor, que progresivamente nos encontraremos con mayor participación china en nuestros mercados tradiciones y que como no podemos «ponerle puertas al campo», en muchos casos es mejor llegar a un entendimiento que a un enfrentamiento, siempre y cuando el beneficio sea mutuo. 